\title{
Recent progress in SDM amplifiers
}

\author{
Yongmin Jung*, Qiongyue Kang, Saurabh Jain, Shaif-ul Alam, and David J. Richardson \\ Optoelectronic Research Center, University of Southampton, Southampton SO17 1BJ, UK
}

\begin{abstract}
Space division multiplexing (SDM) utilizing few-mode fibers or multicore fibers supporting multiple spatial channels, is currently under intense investigation as an efficient approach to overcome the current capacity limit of high-speed longhaul transmission systems based on single mode optical fibers. In order to realize the potential energy and cost savings offered by SDM systems, the individual spatial channels should be simultaneously multiplexed, transmitted, amplified and switched with associated SDM components and subsystems. In this paper, recent progress on the implementation of various SDM amplifiers and its related SDM components is presented.
\end{abstract}

Keywords: Space division multiplexing, few mode fibers, optical amplifiers

\section{INTRODUCTION}

Space Division Multiplexing (SDM) has emerged as a potential means to both increase the capacity and reduce the cost per bit in future communications networks [1,2]. Significant technical progress has been made with capacities in excess of $2 \mathrm{Pbit} / \mathrm{s}$ demonstrated by combining the concepts of multicore and few mode fiber technologies [3]. Whilst technically impressive these have been "hero" lab experiments with only little consideration of practical aspects associated with real world deployment of the technology. Nor of the true cost saving potential which, it is assumed, will be derived by improved sharing of components such as routing devices and amplifiers amongst the multiple spatial channels, and through denser transceiver integration. There is thus a great need to explore the true cost saving potential of SDM and to understand better the implications of sharing individual devices amongst multiple channels. In this paper, a review of recent progress on the implementation of various SDM amplifiers and their related SDM components will be introduced. First, we will summarize/classify the state-of-the-art SDM amplifiers and discuss the structure of the amplifier and their optical properties. Secondly, recently developed fully integrated SDM amplifiers and SDM optical isolators will be discussed in detail. Improved sharing of the optical components and significant device integration were achieved in these devices as required to realize the anticipated cost reduction benefits of SDM.

*ymj@orc.soton.ac.uk; phone+44 238059 4095; www.orc.soton.ac.uk 


\section{CURRENT STATE-OF-THE-ART SDM AMPLIFIERS}

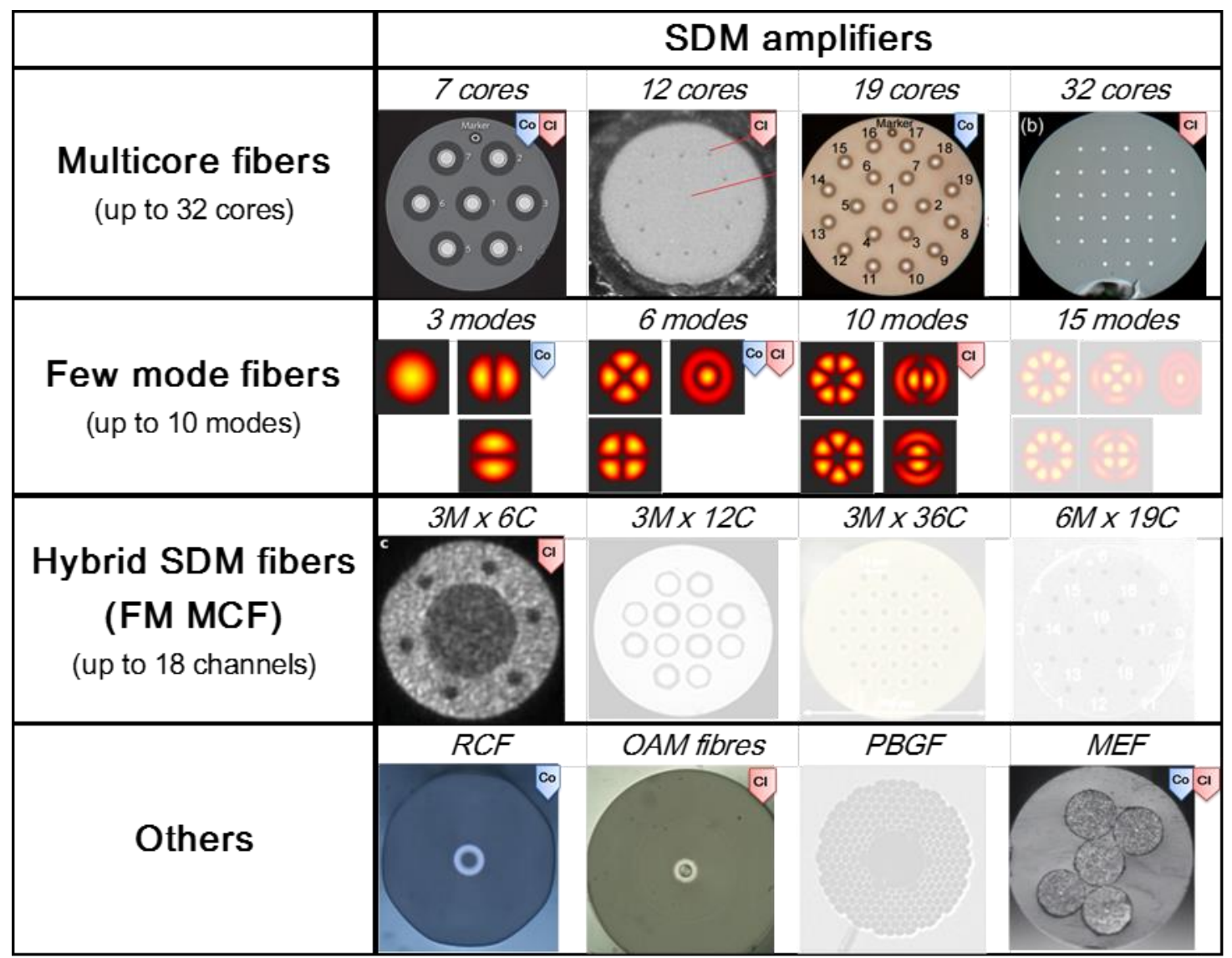

Figure 1. Different types of SDM amplifiers reported so far. Faded images are used to indicate instances where suitable partner amplifier solutions have yet to be demonstrated.

Several different SDM amplifiers have been proposed and investigated to address the particular optical amplification needs of the new generation of SDM transmission fibers. Figure 1 shows the classification of the primary SDM amplifier types reported to date. There are two main SDM amplifiers for achieving multiple spatial channel amplifications within a single fiber: multicore fibers (MCFs) and few-mode fibers (FMFs). In the case of multicore EDFAs (MC-EDFAs), both corepumped and cladding-pumped 7-core EDFAs [4] have been demonstrated in 2011 and 2012, respectively. The corepumped MC-EDFA was then scaled to 19-cores in 2013 [5] and the cladding-pumped MC-EDFA to 32-cores in 2016 [6], which represents the highest core-density MC-EDFA to date. Improved sharing of the optical components and significant device integration were also demonstrated in this 32-core MC-EDFA, as required to obtain the anticipated cost reduction benefits of SDM. For example, two integrated SDM isolators were used to simultaneously prevent unwanted reflections from all 32 cores and two side couplers were used to couple pump beams into the cladding of the active MCF. In the case of few-mode erbium doped fiber amplifiers (FM-EDFAs), both core-pumped [7] and cladding-pumped 6-mode EDFAs [8] were demonstrated in 2014. More recently a cladding-pumped 10-mode EDFA [9] was reported representing the highest mode count FM-EDFA reported so far. The cladding-pumped FM-EDFA, which was originally end-pumped using free space optics [8], has recently been upgraded to incorporate a fully fiberized side-pumping configuration and this functionality makes it possible to construct a fully integrated SDM amplifier capable of providing stable modal amplification without the need for free-space optics. In order to realize a high-capacity hybrid SDM system a hybrid SDM amplifier supporting few-mode multicore fibers represents an essential subsystem and a three-mode, six-core SDM 
amplifier [10] was recently demonstrated supporting 18 spatial channels. Other interesting forms of SDM amplifier, e.g. ring-core fiber amplifiers, OAM fiber amplifiers [11] and MEF amplifiers, have also now been demonstrated.

\section{Fully integrated SDM amplifiers}

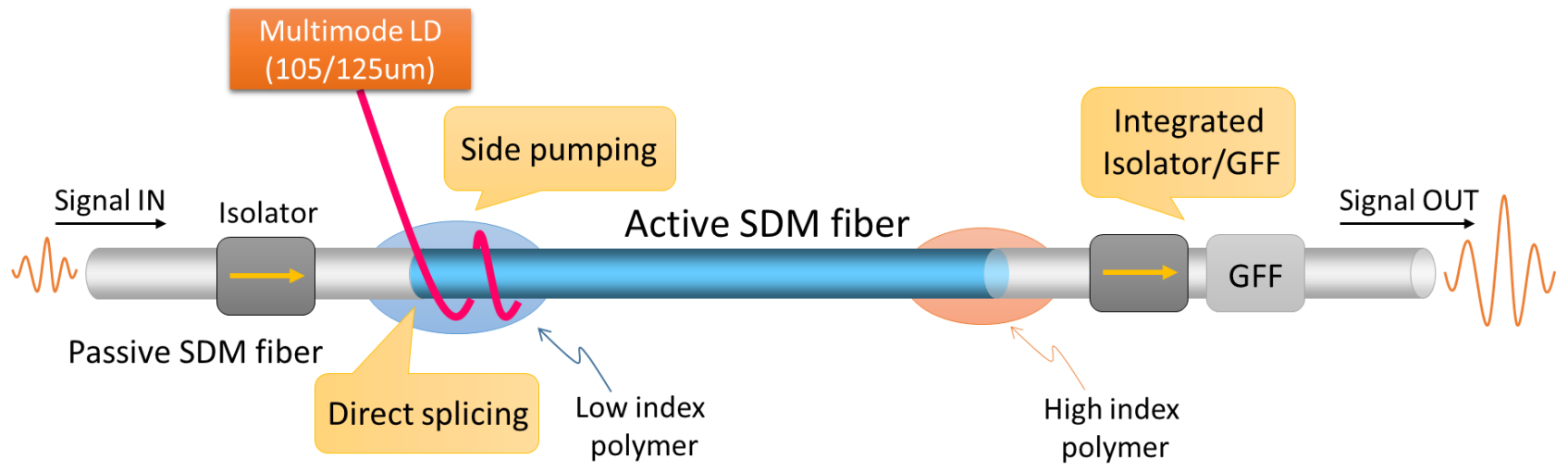

Figure 2. Schematic of a fully integrated SDM amplifier.

Figure 2 shows a schematic of a fully integrated SDM amplifier. As can be seen the configuration, the SDM amplifier looks essentially just like a conventional single mode fiber amplifier, containing input and output optical isolators (which may include optional gain shaping filtering elements if required in the same or a separate package), a WDM pump coupler and a pump diode. The only difference being that all of the in-line components are SDM fiber compatible devices and the pump diode and WDM coupler are multimode devices. Both core and cladding-pumping options are viable methods to realize SDM amplifiers. With regards to pump laser sharing, however, the greatest opportunity is provided by a claddingpumped SDM amplifier configuration. As opposed to core-pumping, which requires multiple high brightness single mode pump diodes and complex (and expensive) means to couple all of the individual pump and signal beams into the individual cores/modes, cladding-pumping allowing the use of just a single high power, but relatively low brightness, multimode pump laser whose output can be fiber-coupled directly into the amplifier in a very straightforward and low-cost manner. The ability to straightforwardly couple the pump radiation into an SDM amplifier is extremely important, particularly if a side coupling approach is used, since it then becomes possible to splice an SDM amplifier directly onto the SDM transmission fiber - opening a route towards fully integrated SDM transmission lines.

\subsection{Side coupler for efficient pump coupling}

The side coupling approach is a convenient way to couple the multimode pump radiation into an active SDM fiber by evanescent field coupling. For efficient side coupling, a passive multimode pump delivery fiber (core/clad diameter $=105 / 125 \mu \mathrm{m}, \mathrm{NA}=0.22$ - compatible with that used on most multimode $976 \mathrm{~nm}$ pump diodes) was tapered down to $15 \mu \mathrm{m}$ with a uniform taper length of $20 \mathrm{~mm}$ and wound with a slight tension around a short $(\mathrm{few} \mathrm{cm})$ stripped length of the active SDM fiber. More than 70\% pump coupling efficiency can be readily achieved with this side coupling method. A UV-curable low-index acrylate polymer can be applied to the tapered section and then cured to ensure robust, stable optical contact within the pump combiner. There is no observable change in pump coupling efficiency as a result of this packaging. Any residual pump power can be removed at the output of the amplifier by applying a high-index polymer to a further section of stripped fiber and removing the associated heat generated. This approach provides a very practical means of pump coupling and is capable of delivering many Watts of pump power into the device if required. Indeed such approaches have allowed coupling of many 100's of Watts of pump power into high power fiber lasers and is indeed already industrially deployed, validating the commercial readiness and suitability of the approach.

\subsection{Fully integrated SDM optical isolators}




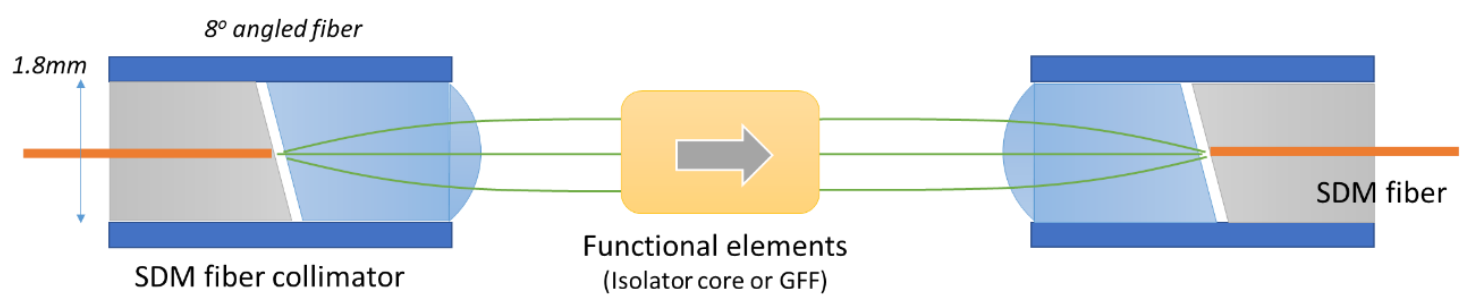

(a)
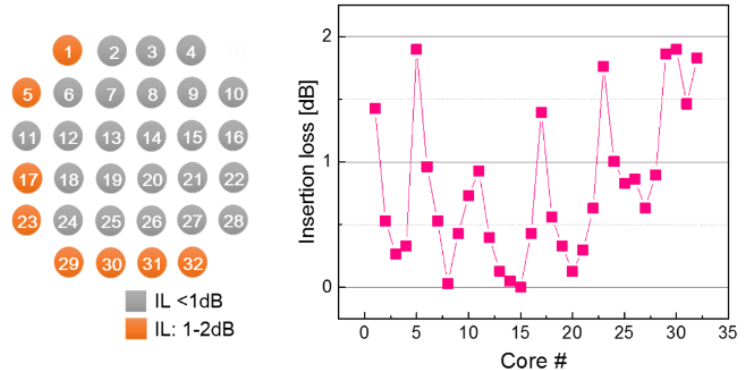

(b)

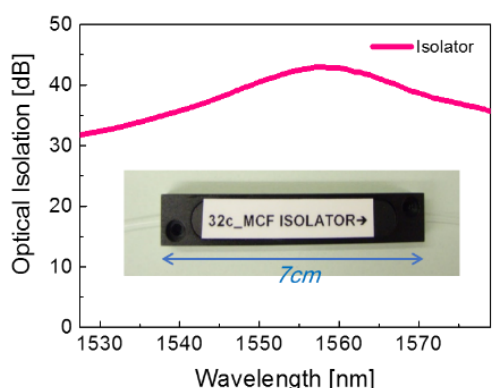

(c)

Figure 3. (a) Schematic of an SDM fiber collimator assembly incorporating various optical functional elements such as optical isolators and gain flattening filters, (b) the measured core-to-core insertion loss variation and (c) optical isolation of the integrated 32-core multicore fiber isolator.

In terms of developing the inline optical isolators and filters required to fulfil our vision of a fully integrated SDM amplifier we have developed a scalable and practical route to manufacturing such components based on the use of optical fiber collimators (i.e. fiber optic collimation and focusing assemblies). This is an important platform already used widely in single mode fiber optic components such as optical isolators, circulators, gain flattening filters, WDM couplers, switches and variable optical attenuators. Compact fiber optic collimators usually use GRIN lens or C-lens elements to transform the emergent light from a single mode optical fiber into a collimated free-space beam that can then be refocused into the another single mode fiber using an second identical assembly in reverse. Optical elements can then be inserted into the free space beam (e.g. a bulk isolator or filter) to provide in-line functionality. Over the past year we have been working to extend this concept to SDM fibers [12, 13], providing an array of new and practical packaged components with functionality and insertion loss comparable to existing equivalent single mode devices.

Figure 3(a) shows a schematic diagram of a representative SDM fiber collimator assembly consisting of a cylindrical rod-type C-lens (or GRIN lens) and a SDM fiber ferrule, fitted in a ferrule sleeve. In order to minimize Fresnel reflection, the SDM fiber was accurately angle-cleaved at an $8^{\circ}$ angle and a C-lens was prepared with both anti-reflection coatings and a complementary $8^{\circ}$ facet angle. Firstly, a C-lens was carefully inserted into the ferrule sleeve and fixed with UV curable adhesive. Then the SDM fiber ferrule was inserted into the other end of the ferrule sleeve and the distance between the C-lens and fiber ferrule was carefully adjusted to achieve a high quality collimated beams. We next developed a compact air-gap device based on two such collimators configured such that we could insert and fix in place different functional elements to provide robustly packaged SDM fiber components. In our recent work [12], we have successfully fabricated a fully packaged 32-core MCF isolator with the optical performance shown in Figure 3(b, c). The average insertion loss of the fabricated isolator was $\sim 0.8 \mathrm{~dB}$ and the core-to-core variation was less than $2 \mathrm{~dB}$. The maximum isolation peak is $43 \mathrm{~dB}$ at $1559 \mathrm{~nm}$ and an optical isolation exceeding $32 \mathrm{~dB}$ was obtained over the full C-band. We also measured the back reflection for the isolator and a value of $-32 \mathrm{~dB}$ was obtained over the full C-band due to the angled fiber facet. The cross-talk between the cores is less than -40dB. This technique can be applicable to other types of SDM fibers and a few mode fiber isolator was also demonstrated in ref. [13].

\section{Fully integrated SDM amplifiers}

\subsection{Fully integrated 6-mode few-mode fiber amplifier}




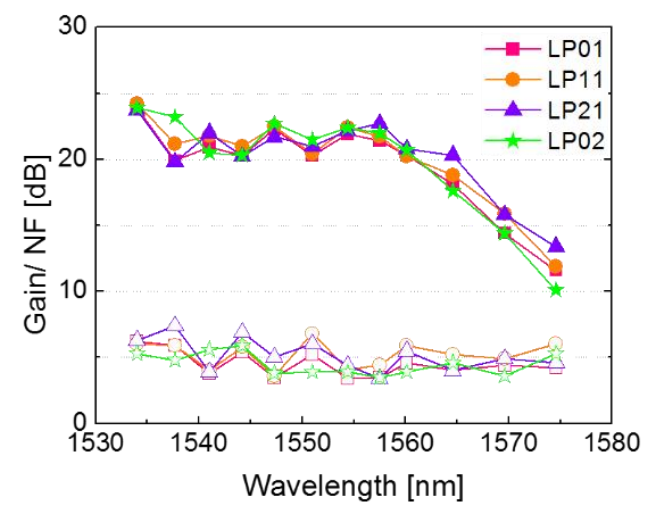

(a)

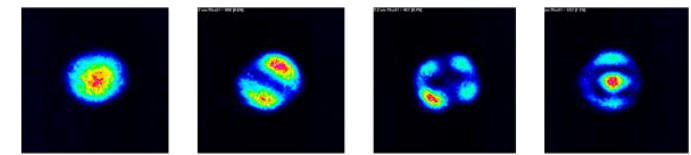

(Amplified signal mode profile)

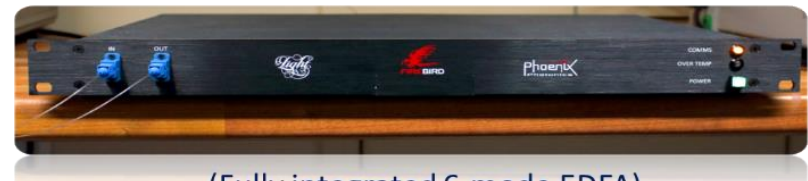

(Fully integrated 6-mode EDFA)

(b)

Figure 4. (a) Measured modal gain/noise figure of the 6-mode FM-EDFA and (b) amplified signal mode profile and the developed rack mountable amplifier module.

With a simple side pump coupler, active SDM fiber and inline components in hand we constructed a few mode fiber amplifier (e.g. 6-mode fiber EDFA supporting $\mathrm{LP}_{01}, \mathrm{LP}_{11 \mathrm{a}, \mathrm{b}}, \mathrm{LP}_{21 \mathrm{a}, \mathrm{b}}$ and $\mathrm{LP}_{02}$ in this demonstration) and performed detailed amplifier characterisation experiments using a phase plate based mode-multiplexer/demultiplexer. Fig. 4(a) shows the gain/NF characteristics of the 6 mode FM-EDFA over the C-band. A $2 \mathrm{~m}$ long 6-mode ring-doped EDF [8] was used as the active medium and 6-mode fiber isolators were included at the input/output of the amplifier (no GFF was used in these particular experiments). Each spatial mode of the amplifier was seeded (for characterisation purposes) with 12 channels distributed over the C-band with an input power of $-7.5 \mathrm{dBm}$ per mode. A small signal gain of $>20 \mathrm{~dB}$ was achieved across the C-band with a differential modal gain of $\sim 3 \mathrm{~dB}$ amongst the mode groups while the averaged noise figure was measured to be between $6-7 \mathrm{~dB}$. The amplified signal mode profiles and fully integrated few-mode amplifier package are presented in Fig. 4(b). This packaged rack mountable amplifier module was developed in conjunction with Phoenix Photonics Ltd. in UK.

\subsection{Fully integrated 32-core multicore fiber amplifier}

The same integration technique can be applied to the MCF amplifier and we have demonstrated a fully integrated 32-core MCF amplifier [6]. The active fiber, 32-core Er/Yb-doped fiber (32c-MC-EYDF), was fabricated based on in-house stackand-draw technique and the multimode pump laser was coupled into the 32c-MC-EDF via side coupling in a co-directional pumping arrangement similar to that of the previous few-mode EDFA. Due to the high core count (i.e. 32 cores), the pump light was quickly absorbed by the doped cores and the population inversion level rapidly reduced along the fiber length. Consequently, in our experiment, two side-couplers were employed, one at the beginning and another in the middle of the active fiber to better balance the population inversion level along the full device length. We also developed an integrated $32 \mathrm{c}-\mathrm{MCF}$ isolator using a micro-lens based fiber collimator assembly. Two such 32c-MCF isolators were spliced at both input and output ends of the 32c-MC-EYDFA and a pair of fan-in/fan-out (FI/FO) devices were incorporated to measure the gain/NF of the amplifier. At a coupled pump power of $16 \mathrm{~W}$, the internal gain and NF of all 32 cores of the amplifier were measured and the results are plotted in Fig. 5(a, b). The majority of the cores exhibited quite similar performance with an average gain of $>17 \mathrm{~dB}$, a NF of $\angle 7 \mathrm{~dB}$ and with a core-to-core variation of 5-6dB. This gain variation is mainly due to the core-to-core insertion loss variation in the passive MCFs (2-4dB), optical isolators (2.5-3.8dB) and splicing imperfections. The inner cores had comparatively less loss variation in the passive components and thus these cores showed more uniform gain and NF performance. It is to be noted however that a few cores ( 6 cores to be exact) exhibit somewhat worse performance than the others. We are in the process of understanding the exact origin of this compromised performance. Nevertheless appreciable gain was achieved for each core, sufficient to allow for high capacity data transmission demonstrated as reported in ref. [6]. 


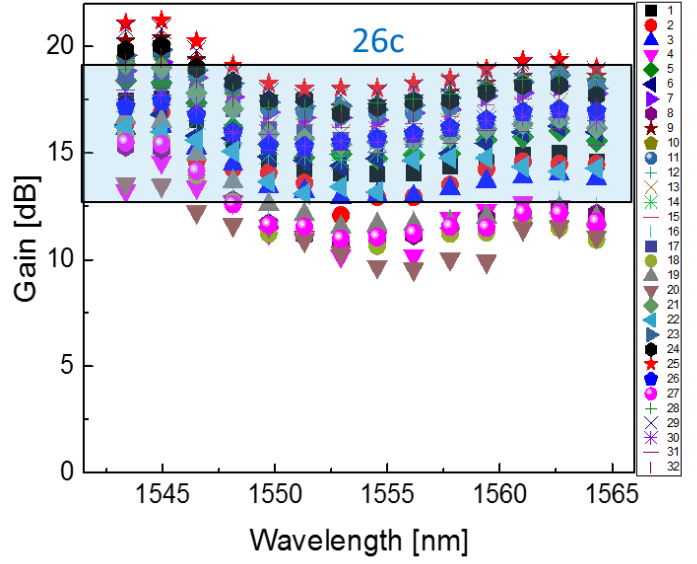

(a)

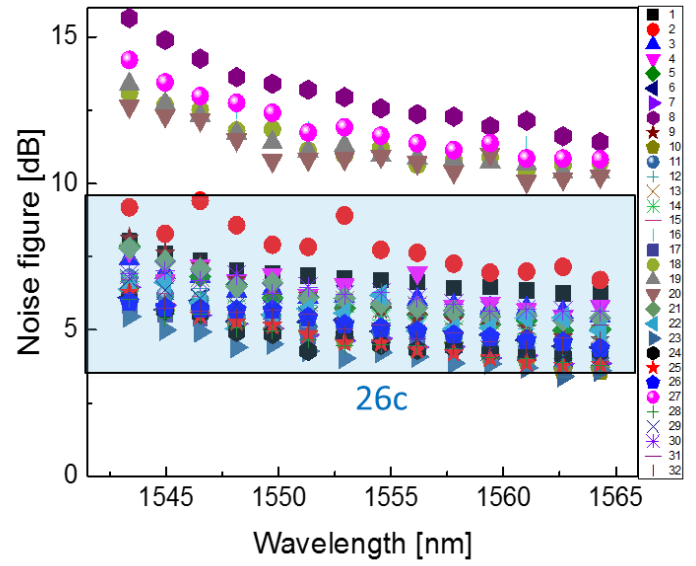

(b)

Figure 5. (a) Measured internal gain and (b) noise figure of the 32-core multicore fiber amplifier.

\section{CONCLUSION}

Over the last few years, significant progress has been made in SDM amplifiers and in-line SDM components. The ability to straightforwardly couple the pump radiation into an SDM amplifier is extremely important, particularly if a side coupling approach is used, since it then becomes possible to splice a SDM amplifier directly onto the SDM transmission fiber opening a route towards fully integrated SDM transmission lines. As an example of the integrated SDM amplifiers, a high spatial density 32-core multicore fiber amplifier was successfully developed in a fully fiberized format and significant component sharing was realized amongst multiple cores. We believe that it is a great improvement in SDM amplifier in terms of number of channels however it is to be appreciated that the amplifier offers limited gain control over independent spatial channel due to the cladding pumping configuration. Future research needs to investigate new ways to control the individual spatial channels to ensure full network functionality.

\section{ACKNOWLEDGEMENT}

Part of this work was supported by the EU-Japan coordinated R\&D project "Scalable And Flexible optical Architecture for Reconfigurable Infrastructure (SAFARI)" funded both by the Ministry of Internal Affairs and Communications (MIC) of Japan and the EU Horizon 2020 Programme.

\section{REFERENCES}

[1] D. J. Richardson, J. M. Fini, L. E Nelson, “Space-division multiplexing in optical fibres,” Nature Photonics 7, 354-362 (2013).

[2] D. J. Richardson, "New optical fibres for high-capacity optical communications Subject Areas: Philosophical Transactions of the Royal Society A, 374, 20140441 (2016).

[3] K. Igarashi, D. Soma, Y. Wakayama, K. Takeshima, Y. Kawaguchi, N. Yoshikane, T. Tsuritani, I. Morita, and M. Suzuki, "Ultra-dense spatial-division-multiplexed optical fiber transmission over 6-mode 19-core fibers," Opt. Express 24, 10213-10231 (2016).

[4] K. S. Abedin, T. F. Taunay, M. Fishteyn, D. J. DiGiovanni, V.R. Supradeepa, J. M. Fini, M. F. Yan, B. Zhu, E. M. Monberg, and F.V. Dimarcello, "Cladding-pumped erbium-doped multicore fiber amplifier," Opt. Express 20, 2019120200 (2012)

[5] Jun Sakaguchi, Werner Klaus, Benjamin J. Puttnam, José Manuel Delgado Mendinueta, Yoshinari Awaji, Naoya Wada, Yukihiro Tsuchida, Koichi Maeda, Masateru Tadakuma, Katsunori Imamura, Ryuichi Sugizaki, Tetsuya Kobayashi, Yusaku Tottori, Masayuki Watanabe, and R. V. Jensen, "19-core MCF transmission system using EDFA with shared core pumping coupled via free-space optics," Opt. Express 22, 90-95 (2014). 
[6] S. Jain, T. Mizuno, Y. Jung, J. Hayes, G. Bai, H. Ono, K. Shibahara, Q. Kang, M. Petrovich, A. Sano, A. Isoda, Y. Miyamoto, Y. Sasaki, Y. Amma, K. Takenaga, K. Aikawa, C. Castro, K. Pulverer, M. Nooruzzaman, T. Morioka, S. Alam, and D. J. Richardson, "32-core inline multicore fiber amplifier for dense space division multiplexed transmission systems," ECOC'16, Th3.A1 (2016).

[7] Y. Jung, Q. Kang, J. K. Sahu, B. Corbett, J. O'Callagham, F. Poletti, S. U. Alam, and D. J. Richardson, "Reconfigurable modal gain control of a few-mode EDFA supporting 6 spatial modes," IEEE Photon. Tech. Lett. 26, 1100-1103 (2014).

[8] Y. Jung, E. L. Lim, Q. Kang, T. C. May-Smith, N. H. L. Wong, R. Standish, F. Poletti, J. K. Sahu, S. U. Alam, and D. J. Richardson, "Cladding pumped few-mode EDFA for mode division multiplexed transmission," Optics Express 22, 29008-29013 (2014).

[9] N. K. Fontaine, B. Huang, z. sanjabieznaveh, H. Chen, C. Jin, B. Ercan, A. Velázquez-Benetez, S. H. Chang, R. Ryf, A. Schülzgen, J. Carlos Alvarado, P. Sillard, C. Gonnet, E. Antonio-Lopez, and R. Amezcua Correa, "Multi-mode Optical Fiber Amplifier supporting over 10 Spatial Modes," OFC'16, Th5A.4. (2016).

[10] C. Jin, B. Huang, K. Shang, H. Chen, R. Ryf, R. J. Essiambre, N. K. Fontaine, G. Li, L. Wang, Y. Messaddeq, S. LaRochelle, "Efficient annular cladding amplifier with six, three-mode cores," ECOC'15, PDP.2.1 (2015).

[11] Y. Jung, Q. Kang, S. Yoo, S. Raghuraman, D. Ho, P. Gregg, S. Ramachandran, S. Alam, and D. J. Richardson, "Optical Orbital Angular Momentum Amplifier based on an Air-Core Erbium Doped Fiber," OFC'16, Th5A.5. (2016).

[12] Y. Jung, S. Alam, Y. Sasaki, and D. J. Richardson, "Compact 32-core multicore fibre isolator for high-density spatial division multiplexed transmission,” ECOC'16, W2.B4 (2016).

[13] Y. Jung, S. Alam, and D. J. Richardson, "Compact few-mode fiber collimator and associated optical components for mode division multiplexed transmission," OFC'16, W2A.40 (2016). 